\title{
AUTOREN Echtzeitfähige Belastungsbestimmung mechanischer Bauteile eines Radladers
}

Lars Brinkschulte,

M. Sc.

st akademischer Mit arbeiter am Institut für

Fahrzeugsystemtechnik

(FAST), Teilinstitut Mobile

Arbeitsmaschinen des

Karlsruher Instituts

für Technologie (KIT) in Karlsruhe.
Durch angepasste Fahrzeugsteuerungssysteme lassen sich die Belastungen und Schädigungen, die Ausfallzeiten und folglich die anfallenden Betriebsund Instandhaltungskosten von mobilen Arbeitsmaschinen reduzieren.

Forscher des KIT haben daher eine Methode zur echtzeitfähigen Belastungsbestimmung am Beispiel der Schwinge eines Radladers entwickelt.
Julian Hafner, M. Sc. ist ehemaliger studentischer Mitarbeiter des Instituts für Fahrzeug systemtechnik (FAST), Teilinstitut Mobile Arbeitsmaschinen und Absolvent des Karlsruher Institut für Technologie (KIT) in Karlsruhe.

Prof. Dr.-Ing. Marcus Geimer ist Institutsleiter des Instituts für Fahrzeugsystemtechnik (FAST), Teilinstitut Mobile Arbeitsmaschinen am Karlsruher

Institut für Technologie (KIT) in Karlsruhe.

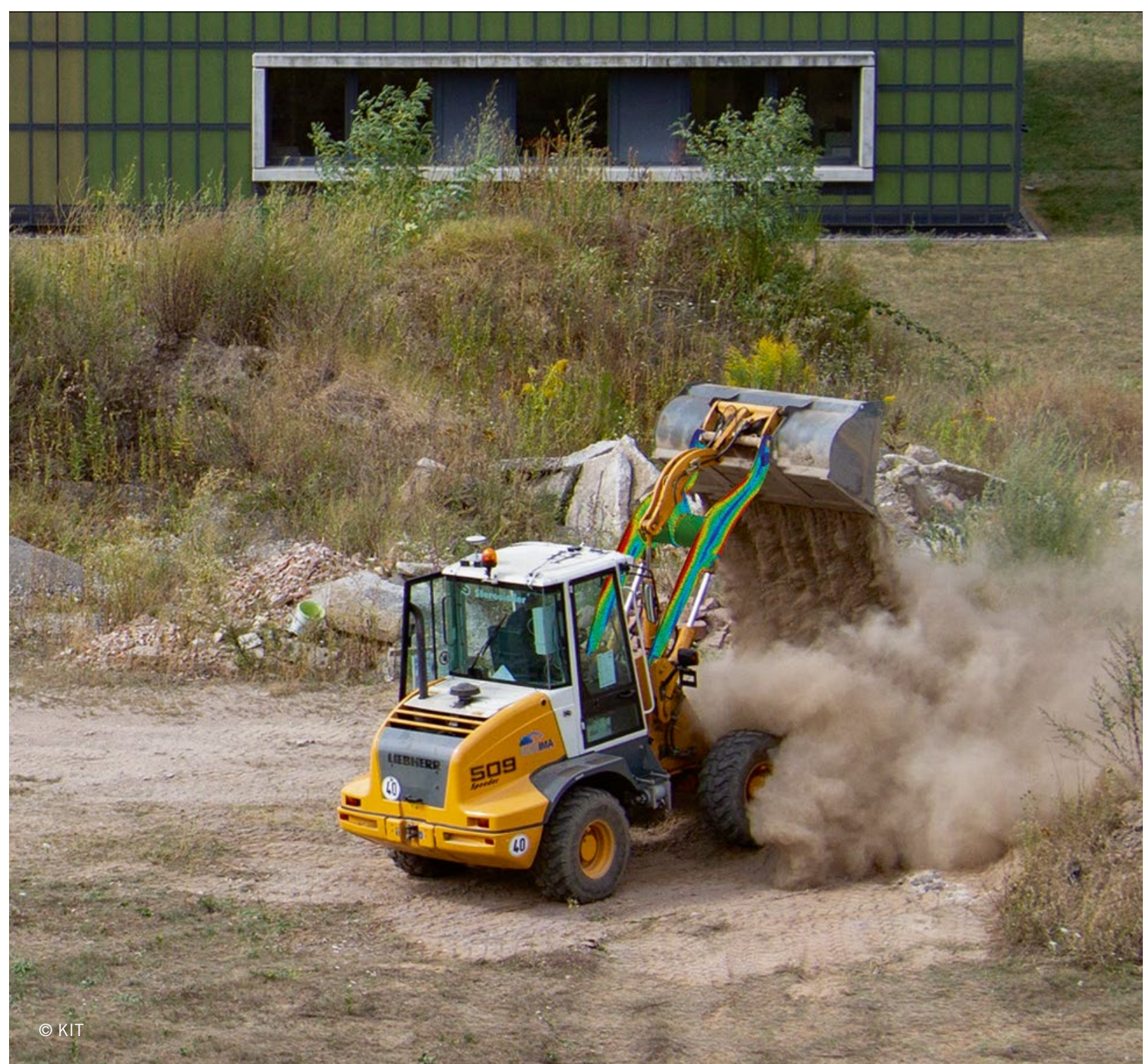


1 EINLEITUNG UND HINTERGRUND

2 METHODE ZUR ECHTZEITFÄHIGEN BELASTUNGSABSCHÄTZUNG

KINEMATISCHE GRUNDBEZIEHUNGEN

SCHÜTTGUTMASSEN - UND KRAFTBESTIMMUNG

SPANNUNGSBERECHNUNG

ZUSAMMENFASSUNG UND ABSCHLUSS

\section{EINLEITUNG UND HINTERGRUND}

Da zur Sicherung von Wettbewerbsvorteilen und Kundenbindung die Lebenszykluskosten von Systemen immer mehr in den Fokus der Maschinenhersteller rücken, ergeben sich Entwicklungsziele wie höhere Verfügbarkeiten, bedarfsgerechte Wartungsintervalle, sowie eine Minimierung von Stillstandszeiten und -kosten.

Auf eine mobile Arbeitsmaschine wirken Belastungen, die mitunter durch die zu erfüllende Arbeitsaufgabe, die Umweltrandbedingungen und den Bediener gekennzeichnet sind. Im praktischen Einsatz haben die Maschinenbediener häufig keine große Erfahrung im Umgang mit der Maschine, beispielsweise beim Einsatz von Fahrzeugen im Baugewerbe. Die Erfüllung der Arbeitsaufgabe hat dabei gegenüber dem Maschinenzustand und der Lebensdauer der Maschine Vorrang. Die Situation verschärft sich durch die Tatsache, dass Unternehmer - gerade im Bereich von Zusatz- und Spezialmaschinen - bevorzugt Mietfahrzeuge zum Einsatz bringen und damit die Bediener ständig mit neuen Fahrzeugen konfrontiert sind. Aufgrund der personenindividuellen Steuerungscharakteristika der Bediener führt dies aus Fahrzeugsicht zu sich ständig verändernden Bewegungsabläufen und Lastsituationen. Die damit im Betrieb häufig auftretende, hohe Belastung kann zu einem vorzeitigen Ausfall der Maschine führen. Dies hat zum einen zur Folge, dass die Tätigkeiten zur Erfüllung der Arbeitsaufgabe nicht weiter verrichtet werden können, zum anderen, dass eine Instandsetzung oder im schlimmsten Fall ein Neuerwerb der Maschine notwendig ist. Beide Auswirkungen resultieren in einem wirtschaftlichen Schaden für den Betreiber.

Die Arbeitsaufgabe und die umweltbedingten Randbedingungen lassen sich unter der Zielfunktion, das geforderte Arbeitsergebnis bei gleichzeitiger Minimierung der Komponentenbelastung zu erreichen, häufig nur schwer beeinflussen. Die Bedienung des Fahrzeugs bietet dahingegen einen evidenteren Freiheitsgrad, um Schädigungen zu minimieren. Mit an die äußeren Bedingungen und die Arbeitsaufgabe angepassten Fahrzeugsteuerungssystemen, die den Fahrer und das Fahrzeug zu einem schädigungsärmeren Betriebsverhalten anleiten, können die Belastungen und Schädigungen reduziert und folglich die anfallenden Betriebs- und
Instandhaltungskosten gesenkt werden. Abschließende Konsequenz ist eine höhere Verfügbarkeit der Maschine. Für ein derartiges Steuerungssystem sind Kenntnisse über die aktuell vorliegende Belastung und die Belastbarkeit von Komponenten einer mobilen Arbeitsmaschine von wesentlicher Bedeutung. Die Entwicklung einer Methode zur echtzeitfähigen Belastungsbestimmung am Beispiel der Schwinge eines Radladers ist daher Inhalt dieses Beitrages.

\section{METHODE ZUR ECHTZEITFÄHIGEN BELASTUNGSBESTIMMUNG}

Als Demonstrationsbeispiel der Methode dient die Schwinge eines Radladers mit Stereolenkung, einem Einsatzgewicht von 5,6 t und einer maximalen Nutzlast von 2 t. Die Auswahl der Belastungsabschätzung der Schwinge beruht auf den vermehrt auftretenden Ausfällen durch Fehl- und Überbelastungen derartiger Maschinen, die in [1] wissenschaftlich untersucht wurden. Das Arbeitsgerät ist als Z-Kinematik mit hydraulisch vorgesteuertem Open-CenterKonstantstromsystem ausgeführt.

Die Maschine ist mit Messtechnik für unterschiedlichste Forschungszwecke ausgestattet. Die zur Belastungsbestimmung der Beispielkomponente Schwinge benötigten Sensoren sind in BILD 1 aufgeführt. Die Sensoren 1 bis 4 sind Druckaufnehmer zur Erfassung der Zylinderkammerdrücke von Hub- und Kippzylinder mit einer Messgenauigkeit von \pm 2 bar. Die Sensoren 5 und 6 sind Entfernungsmessgeräte zur Erfassung der Hublängenänderung der Zylinder mit einer Messgenauigkeit von $\pm 15 \mathrm{~mm}$.

Zur Belastungsbestimmung muss auf Basis der Sensorsignale ein Zusammenhang zwischen diesen und der Bauteilbelastung ermittelt werden. Die Spannungen in den hochbelasteten Stellen des Untersuchungsbauteils dienen als Belastungsindikator, BILD 2. Das dabei zugrundeliegende Vorgehen erfolgt in drei Stufen.

In Stufe 1 (Teil 3 dieses Beitrags) werden die kinematischen Grundbeziehungen der zu betrachteten Baugruppe Hubkinematik aufgestellt. Auf Basis analytischer Ansätze werden die Messwerte der Zylinderausfahrlängen $\left(\vec{L}_{\text {cyl }}\right)$ in Winkelbeziehungen $(\vec{Y})$ der sich relativ zueinander bewegenden Komponenten umgewandelt. In Stufe 2 (Teil 4) folgt die Ermittlung der auf die Komponente wirkenden Kräfte; im ersten Schritt durch die Bestimmung des Schüttgewichts in der Schaufel auf Basis der Zylinderdrücke $\vec{p}_{\text {cyl }}$ und der Winkelbeziehungen $\vec{\Upsilon}$, und anschließend durch die

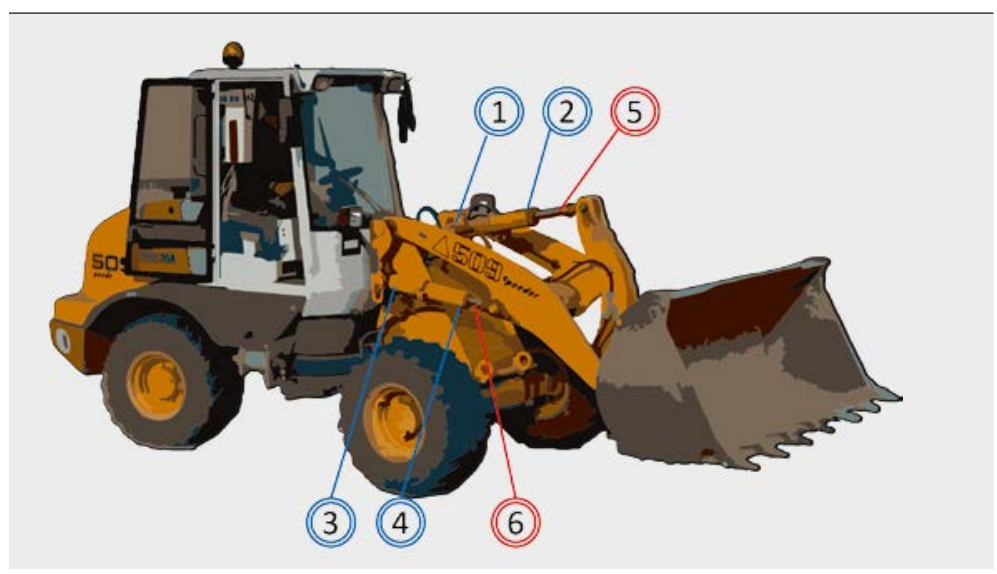

BILD 1 Messtechnik im Fahrzeug (C KIT) 


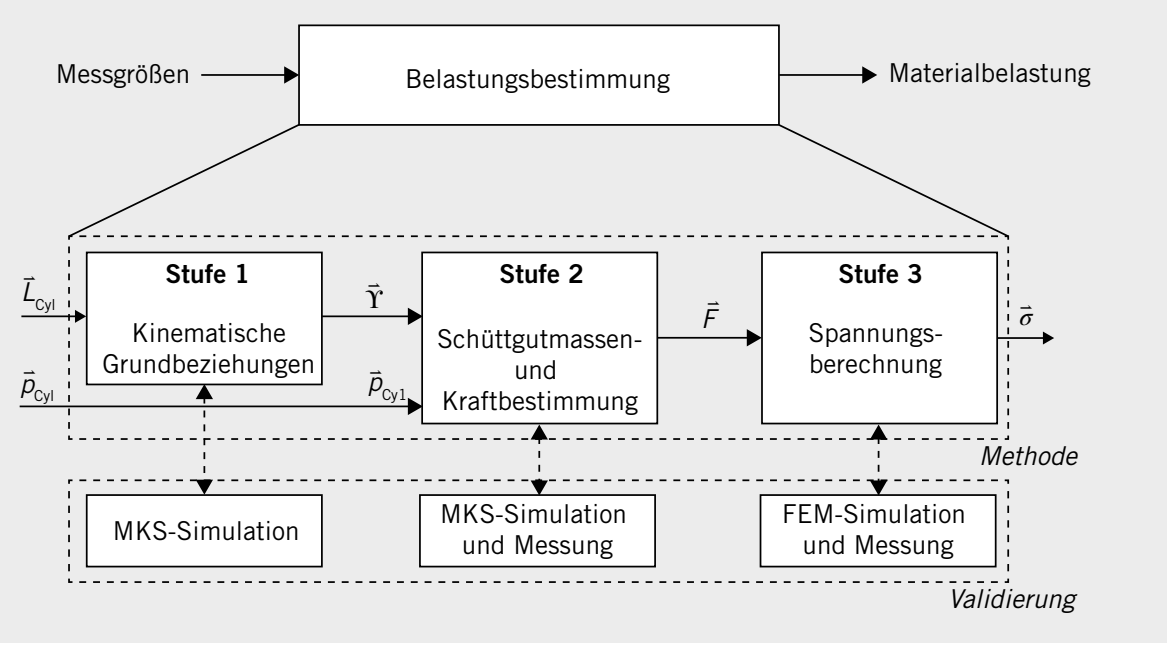

BILD 2 Ablauf der Belastungsbestimmung (@ KIT)

Berechnung aller angreifenden Kräfte $\vec{F}$ in Betrag und Richtung auf Basis der Bewegung und des Schüttgewichts.

Mit Stufe 3 (Teil 5) schließt die Spannungsberechnung der kritischsten Positionen des Bauteils die Betrachtung ab. Auf Basis der Grundlagen der Betriebsfestigkeitslehre oder der Bruchmechanik lässt sich abschließend eine Schädigung der Komponente für die zurückliegenden Arbeitsspiele ermitteln.

Die vorab durchzuführende Parametrierung und Validierung der jeweiligen Algorithmen erfolgt durch diverse Simulationstechniken - Mehrkörpersimulation (MKS) und Finite-Elemente-Methode (FEM) - und ergänzende Messungen an einem Versuchsträger. Die einzelnen Teilsysteme der Methode werden im Folgenden detailliert aufgeführt.

\section{KINEMATISCHE GRUNDBEZIEHUNGEN}

Für eine Spannungsberechnung mithilfe von aktuell anliegenden Belastungen muss die Position des Hubgerüsts zu jedem Zeitpunkt bekannt sein. Zur Bestimmung der Winkel und Abstände wird die Tatsache ausgenutzt, dass die Hubkinematik ein Starrkörpersystem ist, das nur über zwei unabhängige Freiheitsgrade verfügt. Damit lässt sich die Position der Hubkinematik durch Messung von zwei Zustandsvariablen eindeutig bestimmen. Aufgrund einer in

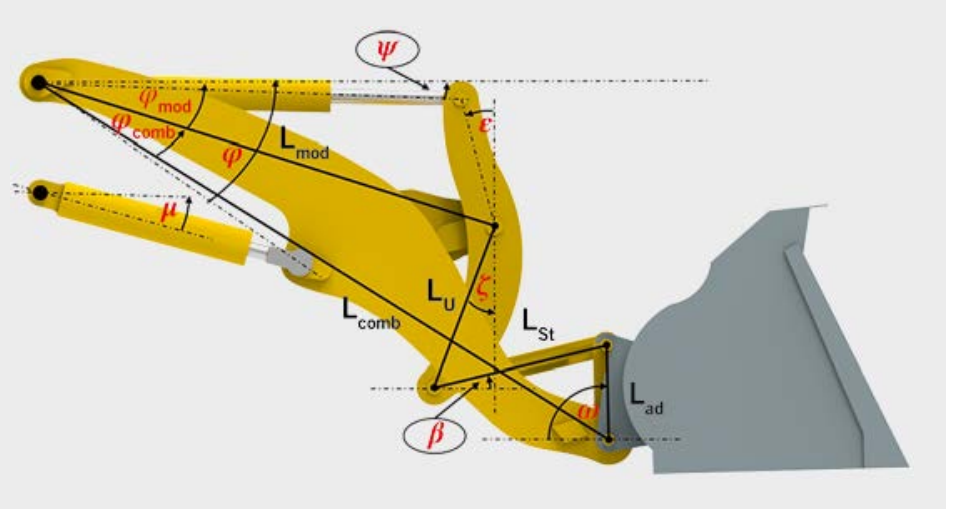

BILD 3 Hubgerüstkinematik (C KIT) der Praxis leicht zu realisierenden Zylinderlängenmessung dienen hierzu die Ausfahrlängen der Hubzylinder und des Kippzylinders. Die neun in BILD 3 dargestellten Winkel sind nichtlinear von den Längen abhängig.

Exemplarisch soll der Zusammenhang am Kippwinkel der Schaufel $\omega$ und dem Winkel der Stange zur Horizontalen $\beta$ gezeigt werden. Das Aufstellen von je einer Gleichung in $x$ - und $y$-Richtung ergibt nach Vereinfachung den Zusammenhang nach GI. 1.

$$
\text { GI. } 1 \text { - } L_{S t}^{2}+\left(\begin{array}{l}
\left(\begin{array}{l}
\sin (\omega) \cdot L_{A d}+\cos (\zeta) \cdot L_{U}-\sin \left(\varphi_{\text {mod }}\right) \cdot \\
L_{\text {mod }}+L_{\text {comb }} \cdot \sin \left(\varphi_{\text {comb }}\right)
\end{array}\right)^{2} \\
+\left(\begin{array}{l}
r_{\text {comb }} \cdot \cos \left(\varphi_{\text {comb }}\right)-\cos \left(\varphi_{\text {mod }}\right) \cdot L_{\text {mod }} \\
-\sin (\zeta) \cdot L_{\text {Div }}-\cos (\omega) \cdot L_{\text {ad }}
\end{array}\right)^{2}
\end{array}\right)=0
$$

Mittels dieser Gleichung kann der Winkel $\omega$ als Funktion der Zylinderlängen ( $L_{\text {Stroke }}$ und $\left.L_{\text {Tilt }}\right)$ dargestellt werden. Dabei gilt: $\zeta=f\left(L_{\text {Stroke }}, L_{\text {Tilt }}\right)$ und $\varphi=f\left(L_{\text {Stroke }}, L_{\text {Tilt }}\right)$ woraus folgt, dass $\left.\omega=f_{(}^{\prime} L_{\text {Stroke }}, L_{\text {Tilt }}\right)$.

Die übrigen vorkommenden Abmaße sind Konstanten und lassen sich aus den Geometriedaten bestimmen. Zur Lösung der Gleichung kommt das nichtlineare Lösungsverfahren Trust-Region [2] zur Anwendung.

Aufgrund der für eine nichtlineare Berechnung notwendigen hohen Rechenkapazitäten werden die Winkel über den gesamten Hub- und Kippbereich vorab bestimmt. Im realen Betrieb werden die Winkel für die aktuell vorliegenden Zylinderlängen durch eine lineare Interpolation zwischen den vorab bestimmten Werten approximiert. Validiert wurde dieses vereinfachte Modell durch die Abbildung der kompletten Kinematik in kommerziellen MKS-Software-Tools.

\section{SCHÜTTGUTMASSEN - UND KRAFTBESTIMMUNG}

Die Grundlage für die in Stufe 3 (Teil 5) vorgestellte Spannungsberechnung sind die auf die Schwinge wirkenden Kräfte. Bekannt sind die Gewichtskräfte der verschiedenen Bauteile und die aus den Drücken der Zylinderkammern resultierenden Kräfte an den Hydraulikzylindern. Auch die Positionen der Gewichtskräfte 


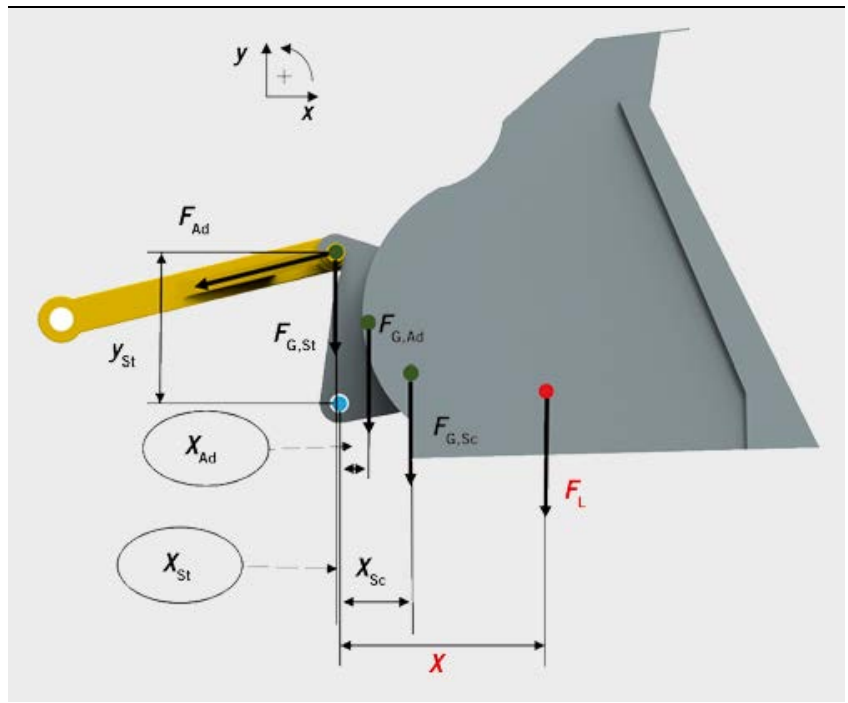

BILD 4 Kräftefreischnitt an Schaufel (@ KIT)

sind durch die in Stufe 1 (Teil 3) bestimmten Winkel vollständig bekannt.

Die Kräfte, die an den Lagerstellen auf die Schwinge wirken, weisen zusätzlich eine signifikante Korrelation mit der in der Schaufel transportierten Masse auf. Für die Berechnung dieser unbekannten Masse im statischen Sonderfall kommen zwei Momentenbilanzen mit je zwei Variablen zur Anwendung. Für die erste Bilanz werden zunächst die Schaufel mit dem Adapter von der Stange und Schwinge freigeschnitten und ein Momentengleichgewicht um die Verbindung zur Schwinge aufgestellt. BILD 4 zeigt die relevanten Kräfte und Abmaße für das um die Aufnahme (blauer Punkt) aufgestellte Gleichgewicht. Unbekannte Größen dieses Gleichgewichts sind die Massenkraft $F_{L}$ und der zugehörige Hebelarm $x$, Gl. 2.

\section{GI. 2}

$F_{\mathrm{L}} \cdot x=\mathrm{L}_{\mathrm{Ad}} \cdot \sin (\omega+\beta) \cdot F_{\mathrm{Ad}}-F_{\mathrm{G}, \mathrm{Ad}} \cdot x_{\mathrm{Ad}}-F_{\mathrm{G}, \mathrm{Sc}} \cdot x_{\mathrm{Sc}}+F_{\mathrm{G}, \mathrm{St}} \cdot x_{\mathrm{St}}$

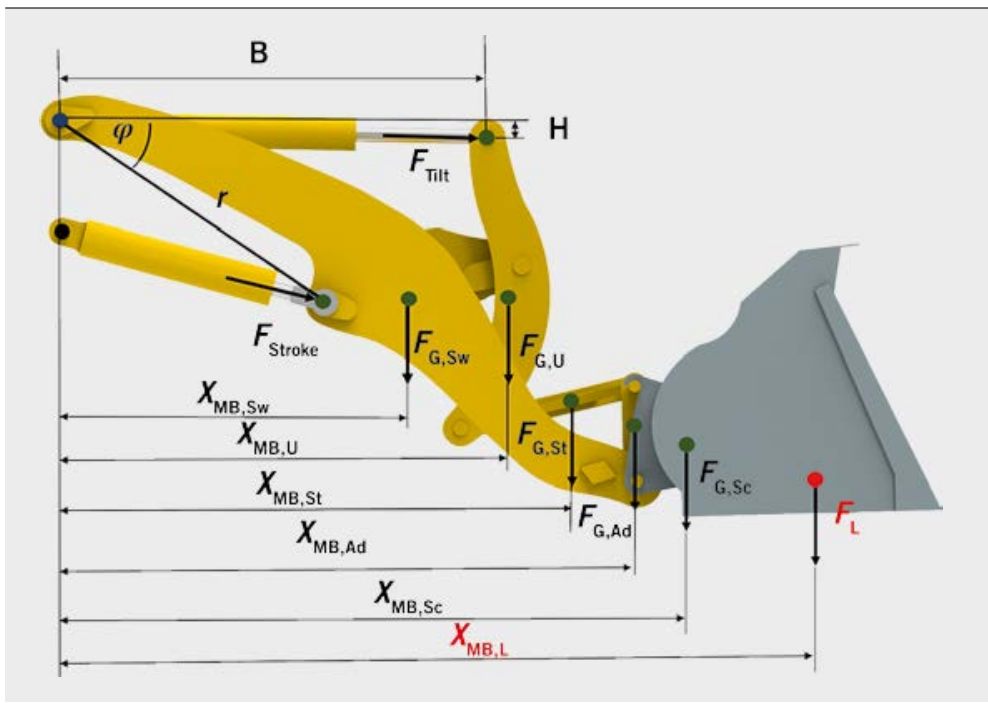

BILD 5 Kräftefreischnitt an Hubgerüst (@ KIT)

Die Kraft $F_{\text {Ad }}$, die von der Stange auf die Schaufel wirkt, kann mithilfe der Drücke am Kippzylinder, der daraus resultierenden Zylinderkraft und dem Hebelgesetz am Kraftumlenker bestimmt werden.

Der Freischnitt der Hubkinematik als Ganzes dient zur Aufstellung der zweiten Momentenbilanz um den Lagerpunkt der Schwinge zum Vorderwagen der Maschine, BILD 5. Die Kombination der beiden Gleichgewichte ermöglicht eine eindeutige Berechnung der Lastkraft des Schüttguts in der Schaufel und somit der Schüttgutmasse, GI. 3.

$$
\begin{aligned}
& \text { GI. } 3 \\
& F_{\mathrm{L}}=\frac{1}{L_{\text {comb }} \cdot \cos \left(\varphi_{\text {comb }}\right)} \cdot\left(\begin{array}{l}
-H \cdot \cos (\psi) \cdot F_{\text {Tilt }}+B \cdot \sin (\psi) \cdot F_{\text {TIIt }} \\
+F_{\text {Stroke }} \cdot r \cdot \sin (\mu-\varphi)-G-F_{\mathrm{L}} \cdot x
\end{array}\right)
\end{aligned}
$$

Für die nachfolgende Bestimmung der Komponentenbelastung ist eine Validierung der Lastkraft des Schüttguts von hoher Bedeu- 


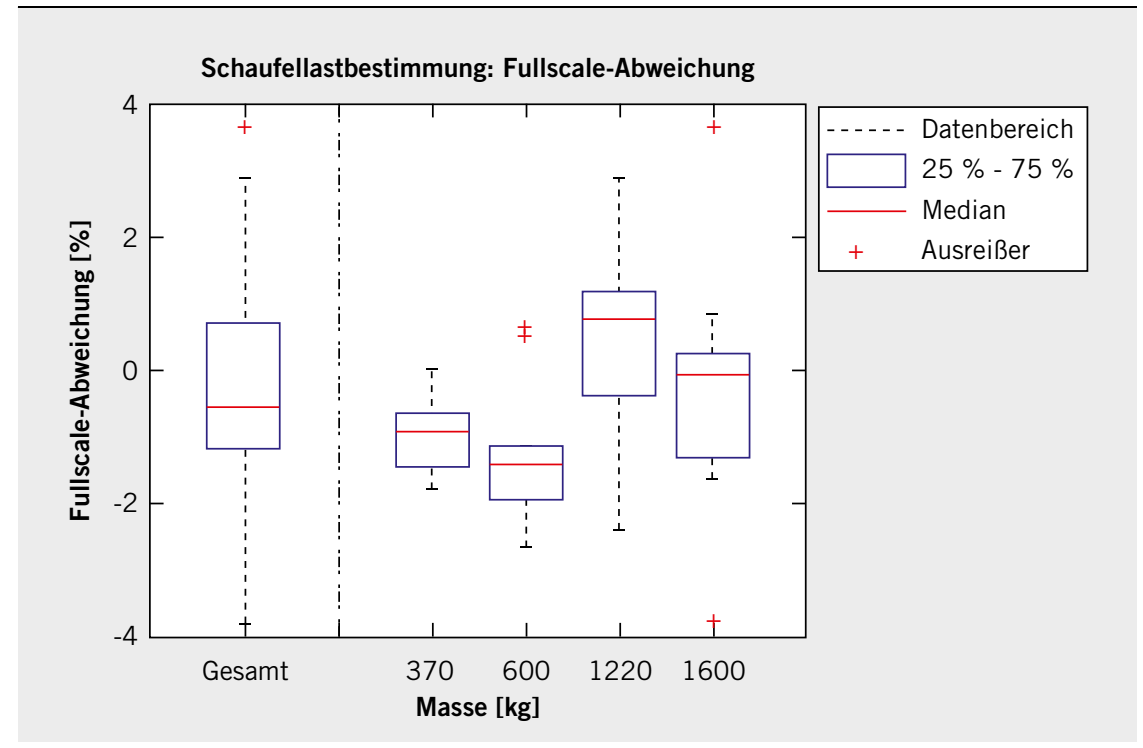

BILD 6 Qualität der Schaufellastbestimmung (C) KIT)

tung. Hierzu wurden umfangreiche Messreihen am realen Versuchsträger durchgeführt; jede beinhaltet Messungen von bis zu 30 sich ändernden Hub- und Kippzylinderstellungen bei konstantem Schüttgutgewicht. Divergente Umgebungsbedingungen wie variierende Außentemperaturen und Schüttgutbeschaffenheiten dienen zur Prüfung der Robustheit des Algorithmus. BILD 6 zeigt die Ergebnisse für die Validierung der Schüttgutmassenbestimmung zu vier über das gesamte Nutzlastspektrum verteilte Messreihen. Dabei sind die Abweichungen als Fullscale(FS)-Abweichungen $e_{\mathrm{m}, \mathrm{FS}}$ dargestellt, also der absolut auftretende Fehler zwischen Berechnung und Messungen ( $m_{\text {calc }}-m_{\text {real }}$ ) geteilt durch die maximale Schüttgutmasse $\left(m_{\max }\right)$ von $2 \mathrm{t}, \mathrm{Gl} .4$.

GI. $4 e_{\mathrm{m}, \mathrm{FS}}=\frac{\left(m_{\mathrm{calc}}-m_{\text {real }}\right)}{m_{\max }}$

Die rote Linie in BILD 6 kennzeichnet den Median. Dies entspricht dem Grenzwert, bei dem genau $50 \%$ aller Werte größer und $50 \%$ kleiner sind. Im Bereich des Kastens - also zwischen dem unteren und oberen Quartil - sind die mittleren $50 \%$ der Abweichungen zu finden. Die Länge der Whiskers (oben, unten) werden auf

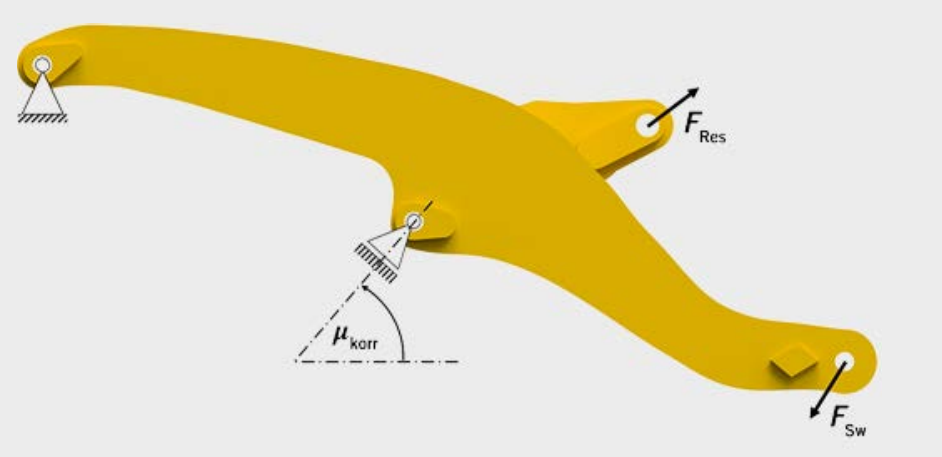

BILD 7 Kräftebilanz für FEM-Belastungsfall (@ KIT) maximal das 1,5-Fache des Interquartilsabstands beschränkt und enden bei dem letzten Datenpunkt innerhalb der Grenzen. Alle Punkte außerhalb der Whisker sind Ausreißer und rot markiert. Es ist erkennbar, dass unabhängig von der geladenen Schüttgutmasse der Median vom realen Messwert um maximal 1,5\% abweicht. $50 \%$ der Messwerte pro Messreihe liegen in einem Toleranzband von maximal $2 \%$. Generell ist zu erkennen, dass die Schüttgutmasse tendenziell unterschätzt wird. Ein masseabhängiger Trend ist nicht zu erkennen. Die Auswertung insgesamt hat gezeigt, dass die größten Abweichungen bei maximalen und minimalen Ausfahrhüben der Zylinder auftreten. Unter Berücksichtigung der Fehlertoleranz und der Reproduzierbarkeit der eingesetzten Sensoren sind die Ergebnisse der Schüttgewichtsbestimmung überzeugend und für den Anwendungsfall ausreichend genau.

Neben der Verwendung der Massenbestimmung zur Belastungsvorhersage, ist ein Nutzen für andere Fragestellungen aus dem Bereich des Maschinenbaus denkbar, zum Beispiel zur Prozesseffizienzbeurteilung oder Baustellenlogistikplanung.

Der vorgestellte Ansatz gilt nur für statische Betriebspunkte also für Betriebspunkte ohne Bewegung der Hubgerüstkinematik. Zur Erweiterung des Ansatzes auf dynamische Betriebspunkte müssen die Beschleunigungen der Massen und deren Trägheiten mitberücksichtigt werden. Außerdem ist bei der unbekannten Massenkraft nicht nur der horizontale, sondern auch der vertikale Hebelarm relevant. Für Schüttgut kann die Form der unbekannten Masse mithilfe der Schaufelform zu einem Trapez approximiert werden. Diese Vereinfachung führt zu einer Massenträgheit und zu einer definierten Schwerpunktposition, die nur von einer Variablen abhängt und somit über die zuvor beschriebene statische Systematik gelöst werden kann. Eine Fahrzeugneigung ist in der Berechnung zusätzlich zu berücksichtigen.

Nach Bestimmung der Schüttgutmasse kann auf die Kräfte in den noch unberücksichtigten Lagerstellen - Verbindung der Schwinge zum Radlader und zum Kraftumlenker - geschlossen werden. Damit sind alle wirkenden Kräfte an der Schwinge bekannt und können zur Erstellung eines Schädigungsmodells in Stufe 3 (Teil 5) genutzt werden. 


\section{SPANNUNGSBERECHNUNG}

Für den statischen Fall kann die Belastung der Schwinge mit dem in BILD 7 dargestellten Modell approximiert werden. Neben den aufgeführten Kräften wirkt zusätzlich das Bauteileigengewicht auf die Schwinge. Für eine dynamische Betrachtung müssen zusätzlich die Beschleunigung der Schwinge und die daraus resultierenden variierenden Beträge der Kräfte an den Lagerpositionen berücksichtigt werden.

Zur Bestimmung der belastungsintensivsten Stellen wird eine vollfaktorielle FEM-Studie durchgeführt. Dabei variieren die Hubund Kippzylinderlängen und zusätzlich die Schüttgutmasse. Der Grabprozess bleibt in der aktuellen Betrachtung unberücksichtigt. Die höchsten Spannungen treten mitunter an den Verbindungsstellen zwischen Schwinge und dem Fahrzeug (Position des Festlagers) und an der Verbindungsstelle zwischen der Schwinge und dem Kraftumlenker (Position der Kraft $F_{\text {Res }}$ ) auf. Die Modellierung der Schwinge erfolgt als Gesamtbauteil, Schweißnähte werden nicht berücksichtigt.

Diese Information dient der Entwicklung eines Belastungsmodells, das im Wesentlichen datenbasiert [3] oder physikalisch motiviert ist. Der für die Verbindungsstellen vorgestellte Ansatz basiert auf einem physikalischen Kerbmodell. Die ermüdungsrelevante Belastung in den spannungsintensiven Stellen der beiden Lager wird als eine Zugnormalspannung mit überlagertem Kerbeffekt angenommen, da die von-Mises-Vergleichsspannung nahezu identisch zur Normalspannung in Richtung der äußeren Kraft ist.

Unter dieser Annahme wird zunächst eine Nominalspannung $\sigma_{\text {nom }}$ berechnet, die sich aus der im Lager anliegenden Kraft und der dazu senkrechten Fläche ergibt. Der im Beispiel dargestellte Kerbfall ist eine Mischung aus einer exzentrischen Bohrung in einer Platte und einer Gelenkkerbe [4]. Für die Kerbfaktoren werden daher zwei Kerbtheorien zugrunde gelegt.

Für diese Kerbfälle gibt es analytische Lösungen, die nur von geometrischen Verhältnisfaktoren abhängen [4]. Für die Theorie der asymmetrischen Bohrung werden nach Peterson [4] die Faktoren $f=\frac{e}{b}$ und $a=\frac{d}{b}$, bei der Theorie der Gelenkkerbe die Faktoren $\frac{c}{H}$ und $\frac{d}{H}$ benutzt. Dabei gibt $H$ die Breite der Platte an, $c$ ist der Abstand des Bohrungsmittelpunkts zur Außenseite des Bauteils in Kraftrichtung und $d$ der Durchmesser der Bohrung.

Der Kerbfaktor $K_{t}$ wird als Subfunktion von allen Faktoren der beiden genannten Kerbfälle dargestellt, GI. 5 .

GI. $5 \quad K_{t}=k_{0}+k_{1} \cdot f+k_{2} \cdot a+k_{3} \cdot \frac{d}{H}+k_{4} \cdot \frac{c}{H}$

GI. 6 zeigt eine Funktion, die auf Basis eines gemeinsamen Kerbspannungsfaktors und der Nominalspannung $\sigma_{\text {nom }}$ eine Maximalspannung $\sigma_{\max }$ im Lagerpunkt angibt. Zur Bestimmung der Subfunktionsparameter wird eine lineare Regression auf Basis der FEM-Lösungen durchgeführt.

GI. $6 \quad \sigma_{\max }=K_{t}\left(f, a, \frac{d}{H}, \frac{c}{H}\right) \cdot \sigma_{\text {nom }}$

Für die Parametrierung der Funktion wird wiederum ein vollfaktorieller FEM-Lösungsraum erzeugt. Die Genauigkeit, die bei der FEM-Lösung erzielt wird, hat signifikanten Einfluss auf die Güte des Kerbmodells. So steigt im Regelfall die Genauigkeit der Ergeb-

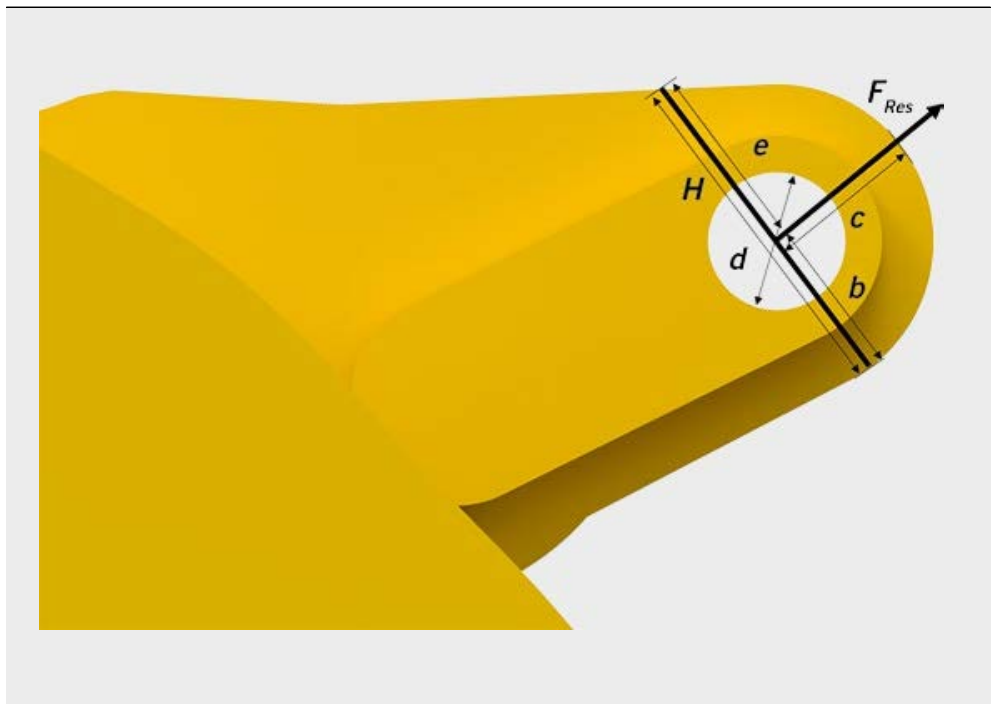

BILD 8 Prinzipdarstellung der Spannungsberechnung (@ KIT)

nisse mit der Anzahl der Knoten, was häufig in einer höheren Spannung im Betrachtungspunkt resultiert. BILD 9 zeigt diesen Umstand am Beispiel eines Lagerauges für einen definierten Lastfall (schwarze Kurve). Mit der Erhöhung der Knotenanzahl steigt zugleich die Rechenzeit (rote Kurve). Eine Steigerung der Rechenkapazität um den Faktor 100 erreicht dabei eine Genauigkeits- und Spannungssteigerung von $17 \%$. Nach zugrunde gelegter linearer Schadensakkumulation nach Miner [5] und der FKM-Richtlinie [6] resultiert daraus für den vorliegenden Belastungsfall eines ungeschweißten Bauteils eine Differenz der ertragbaren Lastspielzahl $N$ und der Lebensdauer von über $140 \%$ (blau), Gl. 7.

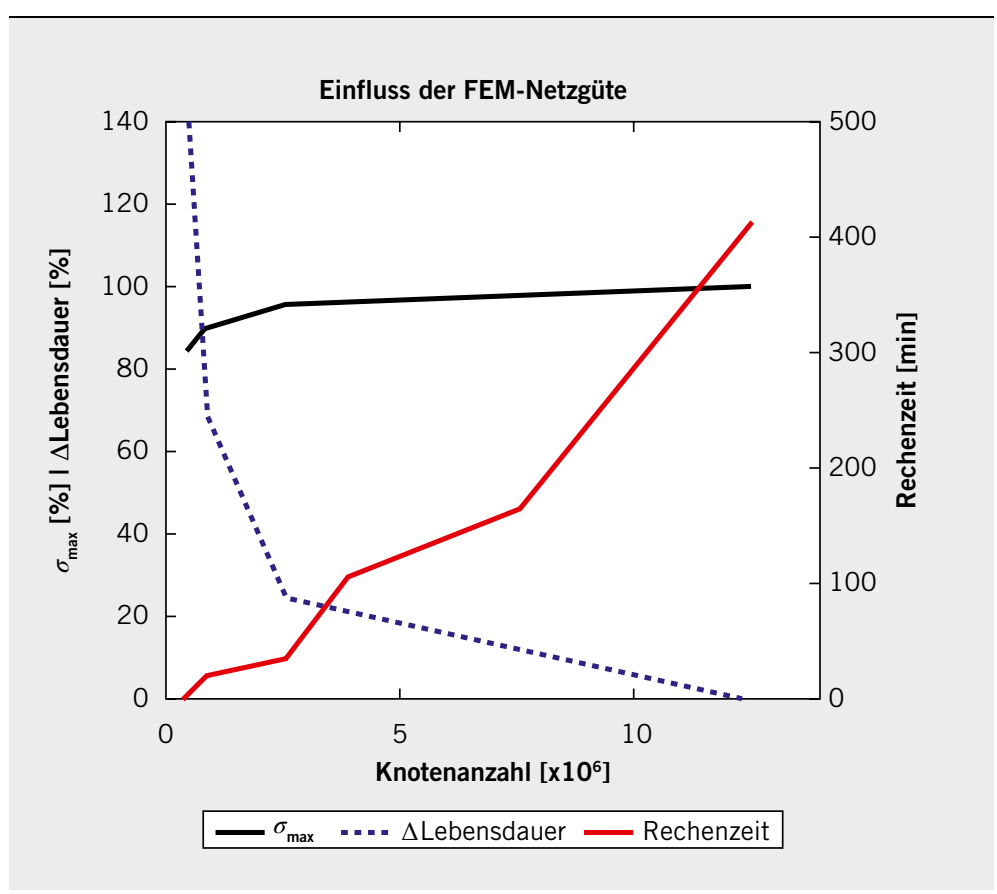

BILD 9 Einfluss der FEM-Netzgüte auf Genauigkeit und Rechenzeit (@ KIT) 


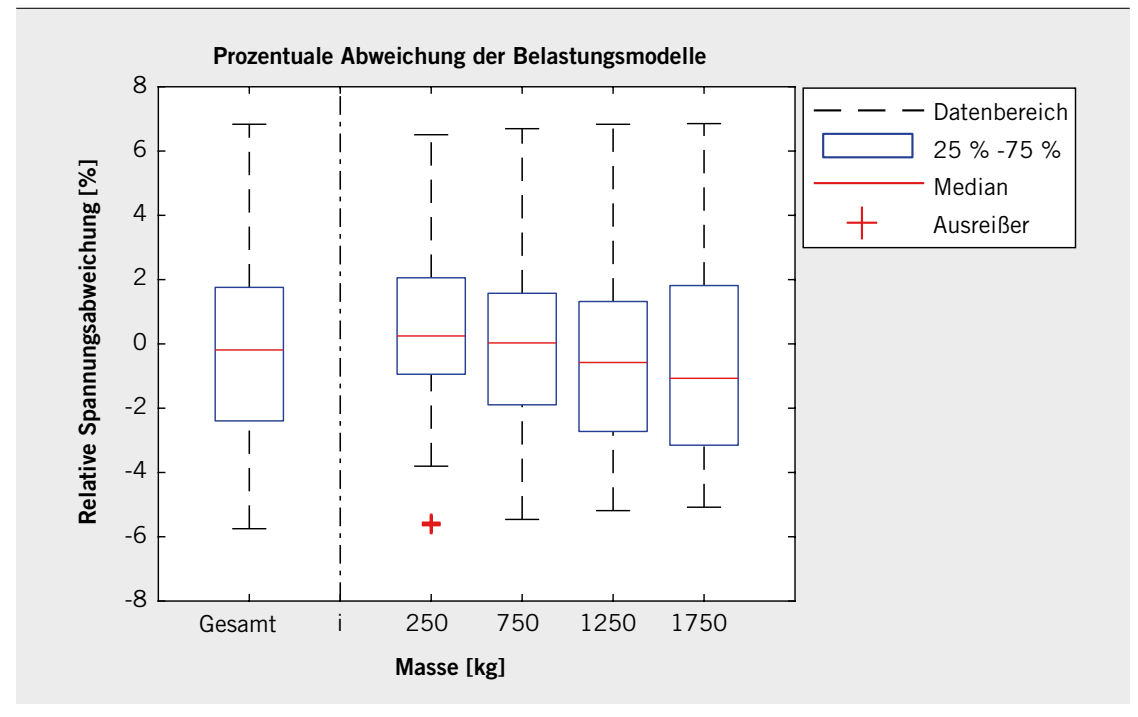

BILD 10 Modellgüte der vereinfachten Belastungsmodelle (C KIT)
GI. $7 \quad\left(\frac{\sigma_{\max , 1}}{\sigma_{\max , 2}}\right)^{5}=\frac{N_{1}}{N_{2}}$

Als Referenzpunkt der Betrachtungen wird das dargestellte feinste Netz mit mehr als $12 \times 10^{6}$ Knoten gewählt. In jedem Betriebspunkt der vollfaktoriellen FEM-Lösung können die nominelle Spannung und alle Geometriekenngrößen bestimmt werden. Dieser Datensatz wird für die lineare Regression der Kerbfunktion verwendet. Zur Auswertung der Theorie wurde ein Modell der Schwinge mit 2,5 $\times 10^{6}$ Knoten verwendet.

BILD 10 zeigt in Form eines Boxplots die relative Spannungsabweichung für den Vergleich der beiden Modelltypen am Beispiel des Lagerauges. Dargestellt sind Lastfälle mit vier verschiedenen Schüttgutmassen bei variierenden Zylinderausfahrhüben, die nicht für die Parametrierung verwendet wurden. Als Vergleich wurde hier die relative Spannungsabweichung $e_{\sigma}$, definiert durch die Differenz der maximalen Spannungswerte der beiden Ansätze $\left(\sigma_{\mathrm{FEM}}-\sigma_{\text {lin }}\right)$ geteilt durch den Spannungswert der FEM-Berechnung $\left(\sigma_{\mathrm{FEM}}\right)$ :

$$
\text { GI. } 8 e_{\sigma}=\frac{\sigma_{\mathrm{FEM}}-\sigma_{\text {lin }}}{\sigma_{\mathrm{FEM}}}
$$

Der Modellvergleich liefert für das vereinfachte Modell sehr gute Ergebnisse, der absolute Median der einzelnen Simulationsreihen ist kleiner $1 \%$. Mit zunehmender Masse ist ein Trend zu höheren Toleranzbändern zu erkennen, 50 \% der Messwerte liegen in einem Toleranzband von $\pm 3 \%$. Die Rechenzeit für das vereinfachte Modell liegt um mehrere Zehnerpotenzen unterhalb der FEM-Berechnung.

\section{ZUSAMMENFASSUNG UND ABSCHLUSS}

Das vorgestellte Verfahren zur echtzeitfähigen Belastungserfassung bildet für eine Vielzahl von Anwendungen einen Mehrwert, der vorgestellte Anwendungsfall der Schwinge eines Radladers ist lediglich als Beispiel anzusehen. So können jegliche schädigungskritische Komponenten unterschiedlichster mobiler Arbeitsmaschinen durch einen derartigen Ansatz untersucht werden.
Die im Beitrag vorgestellte Methode umfasst drei Teile. In einem ersten Schritt erfolgt die Ermittlung von kinematischen Grundbeziehungen. Im zweiten Schritt wird auf Basis der Zylinderhübe und -drücke eine Abschätzung der Schaufelmasse durchgeführt. Die erreichte Genauigkeit beträgt $2 \%$ Fullscale und stellt für die eingesetzte Messtechnik ein sehr gutes Ergebnis dar. Die Kenntnis über die Schüttgutmasse ermöglicht eine Berechnung sämtlicher äußerer- und dynamischer Kräfte im Teilsystem der Arbeitskinematik. Von der Kerbtheorie motivierte und durch FEM-Ergebnisse parametrierte Modelle dienen im Anschluss zur Bestimmung der Materialbelastung. Die Genauigkeit der Modelle hängt im Wesentlichen von der Güte der Parametrierungsdaten ab. Hier wurde eine Übereinstimmung der maximalen Spannungen von $\pm 20 \mathrm{MPa}$ erreicht. Ein Einsatz dieser Methode auf Steuerungssystemen mobiler Arbeitsmaschinen ist heute schon denkbar.

Der vorliegende Beitrag hat den Ansatz lediglich für statische und quasistatische Betriebsbedingungen beschrieben. Eine Erweiterung um die im realen Einsatz auftretenden dynamischen Effekte sind neben einer Dehnungsmessstreifen-basierten, experimentellen Validierung der Spannungsberechnung Gegenstand aktueller Forschungsarbeiten.

\section{LITERATURHINWEISE}

[1] Achelpohl, E. R.: The Effect of Overloading on Reliability of Wheel Loader Structural Components. Online: https://scholarsmine.mst.edu/cgi/viewcontent. cgi? referer=https://www.google.com/\&httpsredir=1\&article $=3660 \&$ context=doct oral_dissertations, aufgerufen am 26.7.2019

[2] Conn, A. R.; Gould, N. I. M.; Toint, P. L.: Trust Region Methods. Society for Industrial and Applied Mathematics, Philadelphia, 2000

[3] Brinkschulte, L.; Geimer, M.: Echtzeitfähige Abschätzung der Restlebens dauer von Komponenten. In: ATZoffhighway 10 (2017) Nr. 3, S. 54-61

[4] Peterson, R. E. und Pilkey, W. D.: Peterson's stress concentration factors. Norwich: Knovel, 2003

[5] Haibach, E.: Betriebsfestigkeit - Verfahren und Daten zur Bauteilberechnung. Berlin: Springer Verlag, 2006

[6] Rennert, R., et al.: Rechnerischer Festigkeitsnachweis für Maschinenbauteile aus Stahl, Eisenguss- und Aluminiumwerkstoffen. Frankfurt am Main: VDMAVerlag, 2012 
Karlsruher Institut für Technologie

\section{Repository KITopen}

Dies ist ein Postprint/begutachtetes Manuskript.

Empfohlene Zitierung:

Brinkschulte, L.; Hafner, J.; Geimer, M.

Echtzeitfähige Belastungsbestimmung mechanischer Bauteile eines Radladers.

2019. ATZheavy duty, 12

doi: $10.5445 / / R / 1000099721$

Zitierung der Originalveröffentlichung:

Brinkschulte, L.; Hafner, J.; Geimer, M.

Echtzeitfähige Belastungsbestimmung mechanischer Bauteile eines Radladers.

2019. ATZheavy duty, 12 (4), 64-70.

doi:10.1007/s35746-019-0043-5 